\title{
Smart Control and Monitoring of Bottle Filling System Based on SCADA
}

\author{
Anjali Gupta, Sanjay Maurya, Ajay Kumar
}

\begin{abstract}
The presented system is a smart control and monitoring system for filling bottles using SCADA. The fast advancement in technology is making whole industrial procedures automated. By the automation in the system it becomes easy to fill various bottles within less time period as manual process is very much time consuming and can be inaccurate upto a level. But after the development of an automated system, the system becomes more accurate and takes lesser time for bottle filling. Filling of various type of liquid in bottles is done by mostly in companies such as water, food companies, paramedical, and chemical industries. Manual process for filling the bottles results in the wastage of the liquid products. Thus an automated bottle filling system is required for improving the speed of operation, cost of production and to system reliability. In this paper automation is introduced in the system by using a software 'WonderwareInTouch SCADA' in the 'Raspberry Pi 3 module'.
\end{abstract}

Index Terms - SCADA, Raspberry pi, Bottle filling method, Proximity Sensor, Level Sensor.

\section{INTRODUCTION}

Today, there is a requirement of automatic bottle filling system that overcomes all the drawbacks of manual bottle filling system as water conservation becomes very important aspect. The bottle shouldn't be over filled or under filled. Automation in this system can be introduced by employing PLC and SCADA [1]. A conveyor belt is employed in the system and bottles are place over that in the process. One by one all the bottles are filled means one bottle is filled at a time. At times the outlet container valve the bottles under it coming out. The level for filling water should be set at a point and the valve is unlocked and bottle gets fill. The position of bottles under the valve is identified by a controlling program. The program also discontinues the rotation of motor, unlocks the valve of solenoid, discontinues the motion of conveyor belt and loads the bottle. Afterwards when the bottle gets loaded entirely the valve should be locked and then the belt should be restarted to fill up the succeeding bottle. Some binary sensors have been used in the system as the equipment is opening and closing according to the set point and stimulates the associated switches. In paper [2] the employment of sensors as switches is shown. The present paper relates to liquid filling system wherein the combination of raspberry pi and sensors is used as input of the system and

Revised Version Manuscript Received on 10 September, 2019.

Anjali Gupta, Department of Electrical \& Electronics Engineering, Noida Institute of Engineering and Technology, UttarPradesh, India.(Email :researchnietip@gmail.com)

Sanjay Maurya, Department of Electrical \& Electronics Engineering, :researchnietip@gmail.com)

Ajay Kumar, Department of Mechanical Engineering, Noida Institute of Engineering and Technology, UttarPradesh, India.. (Email: researchnietip@gmail.com) Noida Institute of Engineering and Technology, UttarPradesh, India.. (Email

the output is different valves. This system would help in conservation of water as well as improves accuracy of the system and lessens the time period taken to fill the bottles. In [4] paper, it is described that'SCADA', a software package interrelated with hardware system located at the topmost of the hardware, normally through 'PLCs' (Programmable Logic Controllers), or supplementary hardware modules.

The systems for cooling, power distribution, and ventilation system uses 'SCADA' control [3]. The forthcoming structures of 'Raspberry Pi' that can behave like both personal computer and PLC, therefore proceed at successive step in many areas such as mechanisation, medical and different industries etc., this is an advantage for using Raspberry Pi is explained in paper [4].

The prime objective of the system is to provide a system for filling water in bottles at less time and with accuracy by using 'Raspberry Pi'. The object is to implement a bottle filling system with SCADA for controlling control room online[5]. The system provides better accuracy in filling bottle upto its pre-defined set point and followed by capping the bottle.

\section{LITERATURE REVIWS}

A system for automatic filling of bottle by employing 'PLC' has been proposed in 2015. The functioning of the system is controlled by using 'PLC'. All the bottles are filled at a time simultaneously. In this bottles of same shape, size and weight have been taken. Operation of liquid filling in bottle and then capping the bottle by using 'PLC' along with 'SCADA' has been projected. The system along with 'PLC' permits for filling the bottle upto a required level of bottle with management of the liquid explained in [6]. Whole system can also be supervised by the application of SCADA. The drawback of this system is, a few numbers of bottles can be loaded by the system in one minute and the system is slow. In paper [10] is concentrated on an Operative Wireless technology for Industrialised Computerization with the help of Raspberry Pi. Internet technology and embedded devices in joint make embedded web server [10]. It is projected that a progressive system for development supervision by a single board computer whose size is like a credit card called Raspberry Pi in [7]. The system is intended by using 'RS232' and a microcontroller which calculates and manages many global constraints. It is discussed the designing and Application of RTU for Observing Weather by using 'RS485' and Raspberry Pi Interfacing [11] 


\section{METHODOLOGY}

The block representation of automatic system for filling liquid in bottle by the use of 'WonderwareInTouch SCADA' is illustrated in figure 1.

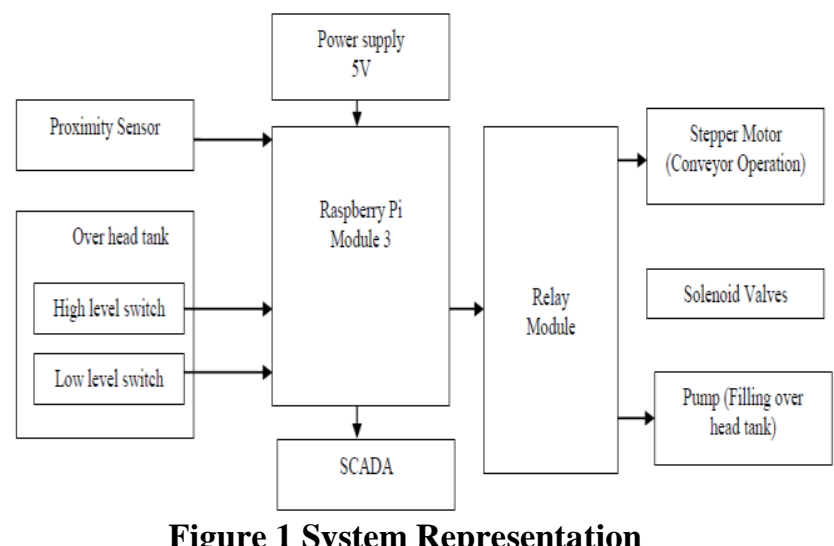

\section{A. Raspberry pi:}

It is a single board computer whose size is similar to the size of a credit card and it has been developed by Raspberry Pi foundation [8][14]. In the presented paper, a Raspberry Pi $2 \mathrm{~B}$ model has been employed. There are forty GPIO pins in the model for interacting with peripherals.

\section{B. Proximity Sensor}

A proximity sensor is employed in the system for identification of an object without any physical contact. The sensor is based on electromagnetic effect and releases electromagnetic beams for identifying the deviations in the field or the deviation in back signal.

\section{Relay}

A switch which is electrically operated is a relay. When the current directs through the relay's coil a magnetic field would be induced to attract a lever and for swapping between contacts. The current flowing through the coil can be in on/off position, thus relays comprises 2 switch points and they're dual changeover buttons. The relays permit single circuitry to change a 2 nd circuitry which is entirely separated from 1 st one.

\section{Solenoid Valve}

This is an electromechanical valve which has been used in the system. The control of the valve is done by an electric current passing by the solenoid. The drifting would be $\mathrm{ON}$ or OFF when there is a 2-port valve, but if there is a 3-port valve, the overflow is swapped between 2 opening ports.

\section{E. Level Detector Sensor}

Level detector sensors identifies the level of various types of liquids, various fluids, different fluidized solids plus slurries, powders and granular materials that show an higher open surface. Ingredients which drift grow into fundamentally horizontal in the respective containers (or different physical limits) due to the gravity while maximum solids pile at a peak angle.

\section{F. Stepper Motor}

A stepper motor which is also named as step motor and stepping motor used in the system is a brushless DC motor which splits a complete revolution into a different numbers of equivalent steps. An instruction to the motor can be given for moving and holding on 1 out of these several equal steps in the absence of feedback sensor (called as an open-loop controller), on condition that the motor is sensibly sized according to the application with respective torque and related speed.

A 'Raspberry pi 3 module' and a personal computer are included by hardware of the system. The operation of conveyor having bottles is done by the motor. For the identification of bottle the proximity sensor has be employed which helps in detecting metal. Two switches one is high level and another is low level are comprised by the overhead tank for setting the level. When the level water drives below as compared to the lowest level switch the motor pumps means switch gets $\mathrm{ON}$, and when the level of water drives above the level of higher level switch for this the motor gets OFF. After the on operation of the motor, the conveyor belt begins the rotation and then the bottle has been detected by the proximity sensor. Now, the solenoid valve starts its function that is filling the bottle for a pre-specified duration and the same process goes on further for each and every bottle. Sensor's output has been applied to the pin of raspberry pi module and then followed by the output result of Raspberry pi module is injected to the connected relay for changing the voltage and driving the output equipment(s) like a stepper motor, pump, valve and solenoid. The identical process was realised in the 'SCADA'.

\section{RESULTS AND DISSUCSSION}

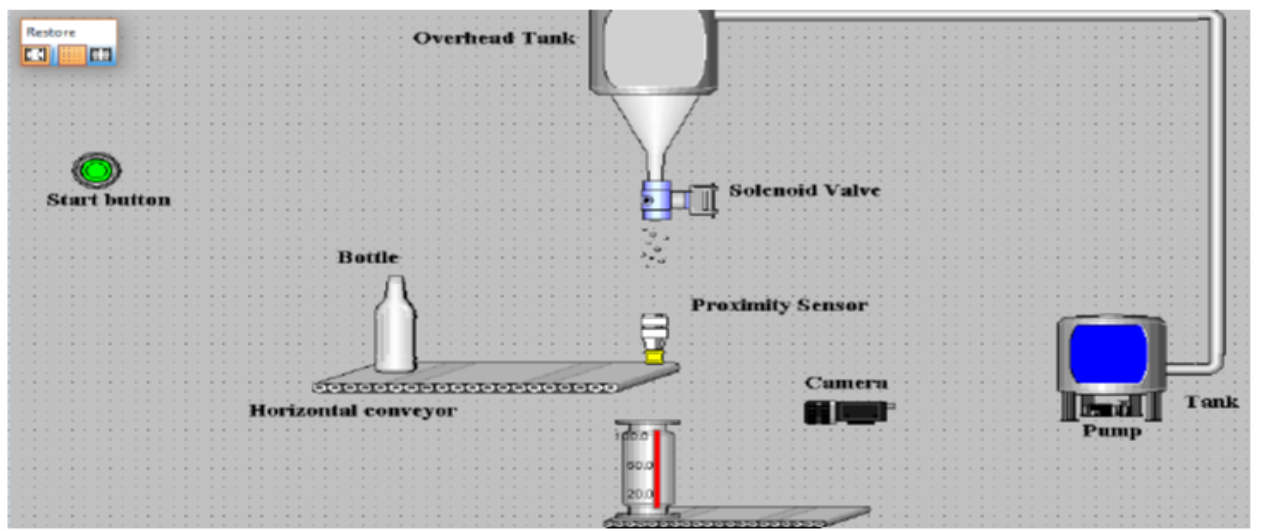

Figure 2 InTouch window maker 
The first step of the process is configuring the Raspberry pi then second step deals with evolving the 'SCADA' and generating the calligraphy for process. The 'SCADA' scheming is established on 'wonderwareInTouch' one after other steps of the procedure.

\section{A. WonderwareIntouch}

SCADA is typically terminated on a software called 'Wonderware'. It is more consistent package that is industrialised by Invensys. It makes it easy to function and this is an open stand for several retailers. The 'Intouchwonderware' 10 is the newest version.

\section{B. Intouch Window Maker}

A progress environment InTouch window maker has an object-intended graphics are employed for creating animated and a display windows which is touch sensitive. These type of display windows have been associated with industrial input/output systems and another Microsoft Windows applications as illustrated in Figure 2.

\section{Intouch Window Viewer}

It is a run-time environment employed for displaying the graphic windows formed in the Window Maker. Window Viewer completes 'InTouch Quick Scripts' and executes logging of historical data, reporting of data as well as processing of data as represented in Figure 3.

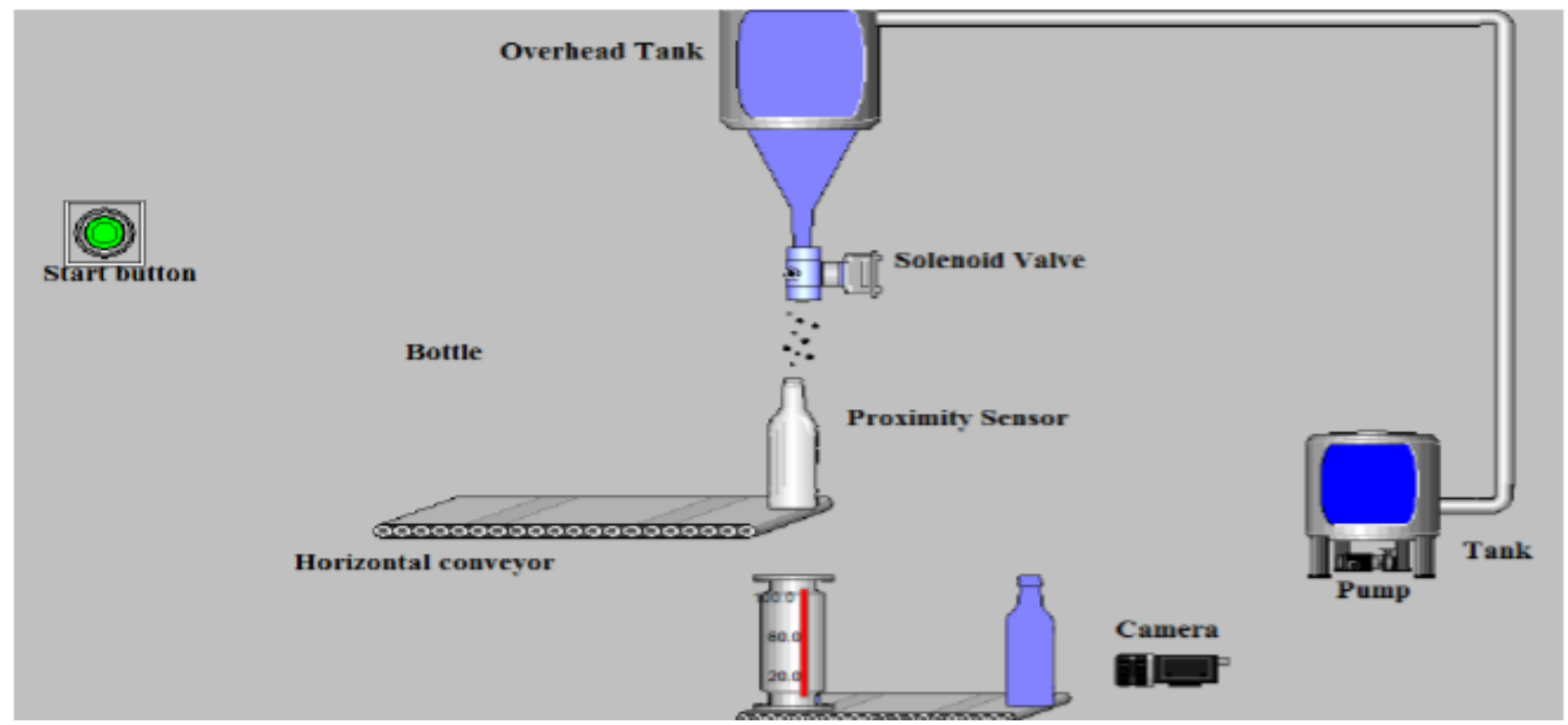

Figure 3 InTouch window viewer

\section{V.CONCLUSION}

An automated system for loading bottles has been proposed in the present paper. The results by the system are discussed in the paper and the proposed algorithm is executed in a better way by the inspection if the bottle is loaded upto a particular level and the amount is appropriate of liquid or not. The system is contactless, accurate and fast. It lessens the complications. By the technique any type of liquid can be loaded. The methodology for the system is very simple and easy for the application.

\section{REFERENCES}

1. S. B. Belekar, A. A. Desai, M. H. Parit, and A. Dakre, "PLC SCADA based Distribution Monitoring \&amp; Control,” Multidiscip. J. Res. Eng. Technol., 2014.

2. M. .Dhwani Parekh; KaushikBhuiya; KilaruLaxmiSahiti; KintaliAnish; Mr. G JoselinRetna Kumar, "Low Cost Wireless Control and Monitoring of Batch Process Using Plc and Scada," Int. J. Sci. Res. Publ., 2013.

3. E. Ozdemir and M. Karacor, "Mobile phone based SCADA for industrial automation," ISA Trans., 2006.

4. H. Ali, A. Ali, R. UlHassnain Syed, A. Khan, and I. Khan, "SCADA Implementation of Industrial Temperature Automation," 2011.

5. C. E. Lin, C. C. Li, A. S. Hou, and C. C. Wu, "A real-time remote control architecture using mobile communication," IEEE Trans. Instrum. Meas., 2003.

6. S. Nadankar, S. Paranjape, G. Pasupuleti, A. Shinde, and
S. Gupta, "IMPLEMENTATION OF BOTTLE FILLING AND CAPPING USING PLC WITH SCADA," Int. Conf. New Era Technol. Sci. Role Manag., 2018.

7. K. Raguvaran and J. Thiyagarajan, "Raspberry PI based global industrial process monitoring through wireless communication," in Proceedings of 2015 International Conference on Robotics, Automation, Control and Embedded Systems, RACE 2015, 2015.

8. S. S. Lagu and S. B. Deshmukh, "Raspberry pi for automation of water treatment plant," in Proceedings - 1st International Conference on Computing, Communication, Control and Automation, ICCUBEA 2015, 2015.

9. RathodRohankumar B., "Design and Implementation of Remote Terminal Unit for Monitoring Weather using Raspberry Pi and RS485 Standard Interface",International Journal for Scientific Research \& Development, vol. 2, no. 03 , pp.435-440,2014.

10. Ganesh S.Thutte and Premraj D, "A Survey of Automatic Detection through SCADA in Network", International Journal of Engineering, Education and Technology (ARDIJEET), Vol.2, No.4,2014.

11. Raguvaran K and Thiyagarajan J., "Raspberry PI Based Global Industrial Process Monitoring Through Wireless Communication", Inter. Conf. on Robotics, Automation, Control and Embedded Systems, pp. 978-81,2015 